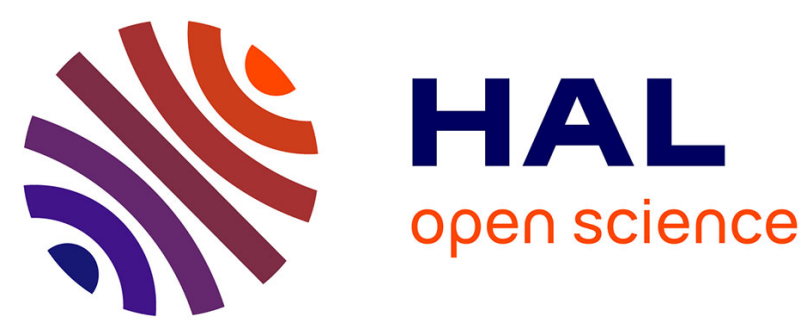

\title{
Design and Analysis of Full-Duplex Massive MIMO Cellular Networks
}

Ertugrul Basar, Miaowen Wen, Raed Mesleh, Marco Di Renzo, Yue Xiao, Harald Haas, Arman Shojaeifard, Kai-Kit Wong, Khairi Ashour Hamdi, Jie Tang

\section{To cite this version:}

Ertugrul Basar, Miaowen Wen, Raed Mesleh, Marco Di Renzo, Yue Xiao, et al.. Design and Analysis of Full-Duplex Massive MIMO Cellular Networks. 2016 IEEE Globecom Workshops (GC Wkshps), Dec 2016, Washington, United States. pp.16693 - 16746, 10.1109/GLOCOMW.2016.7848875 . hal01880096

\section{HAL Id: hal-01880096 https://hal.science/hal-01880096}

Submitted on 15 Jul 2020

HAL is a multi-disciplinary open access archive for the deposit and dissemination of scientific research documents, whether they are published or not. The documents may come from teaching and research institutions in France or abroad, or from public or private research centers.
L'archive ouverte pluridisciplinaire HAL, est destinée au dépôt et à la diffusion de documents scientifiques de niveau recherche, publiés ou non, émanant des établissements d'enseignement et de recherche français ou étrangers, des laboratoires publics ou privés. 


\title{
Design and Analysis of Full-Duplex Massive MIMO Cellular Networks
}

\author{
Arman Shojaeifard*, Kai-Kit Wong*, Marco Di Renzo ${ }^{\dagger}$, Khairi Ashour Hamdi ${ }^{\ddagger}$, and Jie Tang ${ }^{\S}$ \\ ${ }^{*}$ Communications and Information Systems Group, University College London, London, United Kingdom \\ ${ }^{\dagger}$ Laboratoire des Signaux et Systèmes, CNRS, CentraleSupélec, Univ Paris Sud, Université Paris-Saclay, Paris, France \\ ${ }^{\ddagger}$ Microwave and Communication Systems Group, University of Manchester, Manchester, United Kingdom \\ $\S$ Smart Information Processing Centre, South China University of Technology, Guangzhou, China \\ E-Mail: *a.shojaeifard@ucl.ac.uk; *kai-kit.wong@ucl.ac.uk; ${ }^{\dagger}$ marco.direnzo@12s.centralesupelec.fr; \\ $\ddagger$ k.hamdi@manchester.ac.uk; ${ }^{\S}$ eejtang@ @scut.edu.cn
}

\begin{abstract}
This paper provides a theoretical framework for the study of full-duplex (FD) massive multiple-input multiple-output (MIMO) cellular networks over Rician self-interference (SI) and Rayleigh intended and other-interference fading channels. To facilitate bi-directional wireless functionality, we incorporate (i) a downlink (DL) linear zero-forcing with self-interferencenulling (ZF-SIN) precoding scheme at the FD base stations (BSs), and (ii) an uplink (UL) self-interference-aware (SIA) fractional power control mechanism at the FD user equipments (UEs). Linear ZF receivers are further utilized for signal detection in the UL. The results indicate that the UL rate bottleneck in the baseline FD single-antenna system can be elevated by several hundred times via exploiting massive MIMO. On the other hand, the findings may be viewed as a reality-check as the largest spectral efficiency gain from the FD massive MIMO cellular network over its half-duplex (HD) counterpart under state-ofthe-art system parameters is shown to be in the region of $\sim 40 \%$.
\end{abstract}

\section{INTRODUCTION}

Massive multiple-input multiple-output (MIMO) is considered a de facto technology for the emerging fifth-generation (5G) cellular networks [1], [2]. The distinguishing characterstic of this type of deployment - also known as large scale antenna system (LSAS) - is that the base stations (BSs) equipped with hundreds of antennas communicate with multiple user equipments (UEs) over the same time/frequency bins. Massive MIMO cellular networks, via multiplexing multiple terminals and directing power where it is needed, are thus capabe of achieving much higher spectral and energy efficiencies over the existing long-term-evolution (LTE) standards [3].

Another candidate technology for the next generation of wireless systems involves the simultaneous transmission and reception of information over the same radio resources, i.e., full-duplex (FD) communications [4]. This approach has in the past been deemed as practically infeasible due to the overwhelming self-interference (SI) which arises due to the bidirectional functionality. The rapid advancements in the family of techniques used for mitigating SI has however rendered the practical application of FD increasingly more viable, see, e.g., [5] for information on real-time point-to-point FD prototypes.

The large scale adoption of full-duplexing, e.g., in the context of cellular networks, is however not straightforward and largely remains in its infancy. The main challenge lies in the introduction of SI as well as cross-mode interference between downlink (DL) and uplink (UL) functionalities which significantly increases the design complexity compared to conventional half-duplex (HD) systems. This topic has attracted great attention in the research community and there have been many recent works on FD communications in the context of various cellular setups, such as MIMO small-cell network [6], cloud radio access network (C-RAN) [7], and heterogeneous cellular network (HetNet) [8].

A general trend from emerging results with practical system settings is that any spectral efficiency gains from the FD operation over the HD case occur mostly in the DL and that the UL is the main performance bottleneck. For example, the authors in [9] have shown that the FD functionality in the context of baseline single-input single-output (SISO) cellular networks can result in the doubling of the DL rate at the cost of over a thousand-fold deduction in the UL rate. A potential solution can be to exploit massive MIMO for better resilience against SI and cross-mode interference as highlighted in [10] and [11], which focus on a single-cell LSAS scenario.

Motivated by the above, we develop a theoretical framework for the study of FD massive MIMO cellular networks using the Poisson point process (PPP)-based abstraction model. The Rician fading model is utilized in order to capture the characteristics of SI under generalized cancellation techniques. We employ a DL linear zero-forcing with self-interferencenulling (ZF-SIN) precoding scheme at the FD BSs and an UL self-interference-aware (SIA) fractional power control mechanism at the FD UEs. Linear ZF receivers are further used for decoding in the UL. We characterize the system spectral efficiency using a moment-generating-function (m.g.f.)-based approach and provide closed-form expressions for the conditional statistics of the different signals involved.

Notation: A bold uppercase $\boldsymbol{X}$ is a matrix and a bold lowercase $\boldsymbol{x}$ is a vector; the superscripts $T$, $\dagger$, and + are respectively the transpose, Hermitian-transpose, and pseudoinverse operations; $\mathbb{E}_{x}\{$.$\} is the expectation with respect to x$; $\mathcal{F}_{x}($.$) is the cumulative density function (c.d.f.) of x ; \mathcal{P}_{x}($. is the probability density function (p.d.f.) of $x ; \mathscr{P}(x)$ is the probability of $x ; \mathcal{M}_{x}(z)$ is the m.g.f. of $x ;|x|$ is the absolute value of $x ;\|\boldsymbol{x}\|$ is the Euclidean norm of $\boldsymbol{x} ;\|\boldsymbol{X}\|$ is the Frobenius norm of $\boldsymbol{X} ; \mathbf{I}_{(.)}$is the identity matrix; Null(.) cor- 
responds to a null-space; $\mathcal{H}($.$) is the Heaviside step function;$ $\delta($.$) is the Delta function; \Gamma($.$) and \Gamma(.,$.$) are respectively$ the Gamma and (upper) incomplete Gamma functions; $I_{(.)}($. is the modified Bessel function of the first kind; $Q_{(.)}(.,$.$) is$ the generalized Marcum $Q$-function; ${ }_{2} F_{1}(., . ; . ;),.{ }_{2} \tilde{F}_{1}(., . ; . ;$.$) ,$ and ${ }_{0} \tilde{F}_{1}(; ;$.$) are the Gauss, Regularized Gauss, and Regu-$ larized confluent hypergeometric functions, respectively.

\section{SySTEM MODEL}

In what follows, we provide a detailed description of the FD massive MIMO cellular network under consideration.

\section{A. Network Topology and Operation}

We consider a FD cellular setup where the BSs and UEs are respectably deployed on the two-dimensional Euclidean space via independent stationary PPPs $\Phi^{(b)}$ and $\Phi^{(u)}$ with densities $\lambda^{(b)}$ and $\lambda^{(u)}$. Let $Y_{l}$ and $X_{k}$ denote the locations of the $l$-th BS and the $k$-th UE, respectively. The corresponding Euclidean distance is written as $d_{Y_{l}, X_{k}}=\left\|Y_{l}-X_{k}\right\|$. The BSs are assumed to have $N=N^{t}+N^{r}$ antennas where $N^{t}$ and $N^{r}$ are respectively the number of radio frequency (RF) transmit and receive chains. The UEs are in turn assumed to be equipped with single RF transmit/receive chains. In the DL, the BSs are assumed to simultaneously serve $\mathcal{U}\left(\ll N^{t}, N^{r}\right)$ UEs per resource block using linear transmit precoding. Full frequency reuse (as is the case for massive MIMO) is also considered in the UL where the scheduled UEs are assumed to simultaneously transmit to their serving BS per resource block. Linear receive filters are then used for UL signal detection.

\section{B. Channel Model and Statistics}

Let $\boldsymbol{g}_{Y_{l}, X_{k}} \in \mathcal{C}^{1 \times N^{t}}, \boldsymbol{G}_{Y_{l}, Y_{j}} \in \mathcal{C}^{N^{r} \times N^{t}}$, and $\boldsymbol{G}_{Y_{l}, Y_{l}} \in$ $\mathcal{C}^{N^{r} \times N^{t}}$ denote the channel from the $l$-BS to the $k$-th UE, the channel from the $l$-th $\mathrm{BS}$ to the $j$-th BS, and the SI channel at the $l$-th $\mathrm{BS}$, respectively. In addition, $\boldsymbol{h}_{X_{k}, Y_{l}} \in \mathcal{C}^{N^{r} \times 1}$, $h_{X_{k}, X_{i}}$, and $h_{X_{k}, X_{k}}$ are respectively used to represent the channel from the $k$-th UE to the $l$-th $\mathrm{BS}$, the channel from the $k$-th UE to the $i$-th UE, and the SI channel at the $k$ th UE. The SI channels are subject to Rician fading with independent and identically-distributed (i.i.d.) elements drawn from $\mathcal{C N}\left(\mu, v^{2}\right)$. All other channels are assumed to undergo Rayleigh fading with i.i.d. elements drawn from $\mathcal{C N}(0,1)$. We use the unbounded path-loss model with exponent $\beta(>2)$. Perfect channel state information (CSI) is assumed throughout.

\section{Cellular Association Strategy}

By invoking the Campbell-Mecke's theorem, the DL analysis is carried out for an arbitrary UE $k^{*}$ assumed to be located at the center. We consider a cellular association strategy where the $\mathrm{UE} k^{*}$ is exclusively served by a BS $l^{*}$ which provides the greatest received signal power. For homogeneous deployments, this is equivalent to the cell selection approach based on the closest distance. The UL analysis, on the other hand, is carried out for the reference UE at its serving BS. Similarly, all other scheduled UEs in the system are assumed to have been exclusively associated with their closest deployed BS. With a slight abuse of notation, the location of the $k$-th scheduled UE of the BS located at $Y_{l}$ is denoted with $X_{k}^{l}$. For consistency's sake, $X_{k^{*}}^{l^{*}}$ is thus used in place of $X_{k^{*}}$ where the context is clear. The reference transmitter-receiver distance p.d.f. is

$$
\mathcal{P}_{d_{Y^{*}, X^{k^{*}}}^{l^{*}}}(r)=2 \pi \lambda^{(b)} r \exp \left(-\pi \lambda^{(b)} r^{2}\right) .
$$

\section{Baseband Signals Representations}

Let $\boldsymbol{G}_{Y_{l}}=\left[\boldsymbol{g}_{Y_{l}, X_{k}^{l}}^{T}\right]_{1 \leq k \leq \mathcal{U}}^{T} \in \mathcal{C}^{\mathcal{U} \times N^{t}}$ denote the concatenation of the DL channels from the $l$-th BS to its $\mathcal{U}$ UEs. In addition, we use $s_{Y_{l}}=\left[s_{Y_{l}, X_{k}^{l}}\right]_{1 \leq k \leq \mathcal{U}}^{T} \in \mathcal{C}^{\mathcal{U} \times 1}$ to denote the DL complex symbol vector from the $l$-th BS to its $\mathcal{U}$ UEs such that $\mathbb{E}\left\{\left|s_{Y_{l}, X_{k}^{l}}\right|^{2}\right\}=1$. The transmit signal vector with linear precoding at the $l$-th BS is $\boldsymbol{t}_{Y_{l}}=\boldsymbol{V}_{Y_{l}} \boldsymbol{s}_{Y_{l}}$ where $\boldsymbol{V}_{Y_{l}}=\left[\boldsymbol{v}_{Y_{l}, X_{k}^{l}}\right]_{1 \leq k \leq \mathcal{U}} \in \mathcal{C}^{N^{t} \times \mathcal{U}}$ is the precoding matrix. The DL received signal at the reference UE is hence given by

$$
\begin{aligned}
& y_{d}=\underbrace{\sqrt{\frac{p^{(b)}}{\mathcal{U}}} d_{Y_{l^{*}, X_{k^{*}}}^{l^{*}}}^{-\frac{\beta}{2}} \boldsymbol{g}_{Y_{l^{*}}, X_{k^{*}}^{l^{*}}} \boldsymbol{v}_{Y_{l^{*}}, X_{k^{*}}^{l^{*}}} s_{Y_{l^{*}}, X_{k^{*}}^{l^{*}}}}_{\text {intended signal }, d}
\end{aligned}
$$

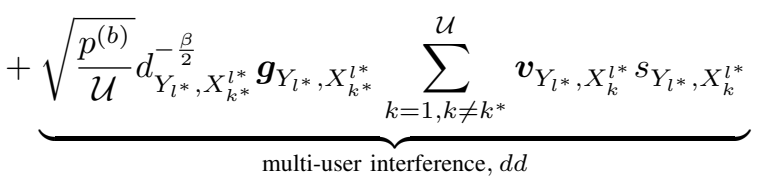

$$
\begin{aligned}
& +\underbrace{\sqrt{\frac{p^{(b)}}{\mathcal{U}}} \sum_{Y_{l} \in \Phi^{(b)} \backslash\left\{Y_{l^{*}}\right\}} d_{Y_{l}, X_{k^{*}}^{l^{*}}}^{-\frac{\beta}{2}} \boldsymbol{g}_{Y_{l}, X_{k^{*}}^{l^{*}}} \boldsymbol{V}_{Y_{l}} \boldsymbol{s}_{Y_{l}}}_{\text {inter-cell interference, } d \rightarrow d} \\
& +\underbrace{\sum_{X_{k}^{l} \in \tilde{\Phi}(u) \backslash\left\{X_{k^{*}}^{l^{*}}\right\}} \sqrt{p_{X_{k}^{l}(u)}^{u}} d_{X_{k}^{l}, X_{k^{*}}^{l^{*}}}^{-\frac{\beta}{2}} h_{X_{k}^{l}, X_{k^{*}}^{l^{*}}} s_{X_{k}^{l}}}_{\text {cross-mode interference, } u \rightarrow d} \\
& +\underbrace{\sqrt{p_{X_{k^{*}}^{l^{*}}}^{(u)}} h_{X_{k^{*}}^{l^{*}}, X_{k^{*}}^{l^{*}}} s_{X_{k^{*}}^{l^{*}}}}_{\text {self-interference, } s i \rightarrow d}+\underbrace{\eta_{X_{k^{*}}^{l^{*}}}}_{\text {noise }}
\end{aligned}
$$

where $p^{(b)}$ is the BS total transmit power, $\tilde{\Phi}^{(u)}$ is the set of scheduled UEs locations, $p_{X_{k}^{l}}^{(u)}$ is the $l$-th BS $k$-th scheduled UE transmit power for sending the complex symbol $s_{X_{k}^{l}}$, and $\eta_{X_{k^{\prime *}}}$ is the complex additive white Gaussian noise (AWGN) at UE $k^{*}$ with mean zero and variance $\sigma^{2}$, respectively. For analytical tractability, we hereafter discard the spatial dependencies and assume $\tilde{\Phi}^{(u)}$ is a stationary PPP with density $\lambda^{(u)}=\mathcal{U} \lambda^{(b)}$.

Next, let $\boldsymbol{H}_{Y_{l}}=\left[\boldsymbol{h}_{X_{k}^{l}, Y_{l}}\right]_{1 \leq k \leq \mathcal{U}} \in \mathcal{C}^{N^{r} \times \mathcal{U}}$ represent the compound UL channel matrix at the $l$-BS from its $\mathcal{U}$ scheduled UEs. The linear receiver filter at the $l$-th BS is denoted using $\boldsymbol{W}_{Y_{l}}=\left[\boldsymbol{w}_{X_{k}^{l}, Y_{l}}^{T}\right]_{1 \leq k \leq \mathcal{U}}^{T} \in \mathcal{C}^{\mathcal{U} \times N^{r}}$. The post-processing UL signal from the reference UE at its serving BS can be written as

$$
\begin{aligned}
y_{u} & =\underbrace{\sqrt{p_{X_{k^{*}}^{l^{*}}}^{(u)} d_{X_{k^{*}}^{l^{*}}, Y_{l^{*}}}^{-\frac{\beta}{2}} \boldsymbol{w}_{X_{k^{*}}^{l^{*}}, Y_{l^{*}}}^{T} \boldsymbol{h}_{X_{k^{*}}^{l^{*}}, Y_{l^{*}}} s_{X_{k^{*}}^{l^{*}}}}}_{\text {intended signal, } u} \\
+ & \underbrace{\sum_{k=1, k \neq k^{*}}^{\mathcal{U}} \sqrt{p_{X_{k}^{l^{*}}}^{(u)}} d_{X_{k}^{l^{*}}, Y_{l^{*}}}^{-\frac{\beta}{2}} \boldsymbol{w}_{X_{k^{*}}^{l^{*}}, Y_{l^{*}}}^{T} \boldsymbol{h}_{X_{k}^{l^{*}}, Y_{l^{*}}} s_{X_{k}^{l^{*}}}}_{\text {multi-user interference, } u u}
\end{aligned}
$$




$$
\begin{aligned}
& \underbrace{\sum_{X_{k}^{l} \in \tilde{\Phi}(u) \backslash\left\{\forall X_{k}^{l^{*}}\right\}} \sqrt{p_{X_{k}^{l}}^{(u)}} d_{X_{k}^{l}, Y_{l^{*}}}^{-\frac{\beta}{2}} \boldsymbol{w}_{X_{k^{*}}^{l^{*}}, Y_{l^{*}}}^{T} \boldsymbol{h}_{X_{k}^{l}, Y_{l^{*}}} s_{X_{k}^{l}}}_{\text {inter-cell interference, } u \rightarrow u} \\
& +\underbrace{\sqrt{\frac{p^{(b)}}{\mathcal{U}}} \sum_{Y_{l} \in \Phi^{(b)} \backslash\left\{Y_{\left.l^{*}\right\}}\right.} d_{Y_{l}, Y_{l^{*}}}^{-\frac{\beta}{2}} \boldsymbol{w}_{X_{k^{*}}^{l^{*}}, Y_{l^{*}}}^{T} \boldsymbol{G}_{Y_{l}, Y_{l^{*}}} \boldsymbol{V}_{Y_{l}} \boldsymbol{s}_{Y_{l}}}_{\text {cross-mode interference, } d \rightarrow u} \\
& +\underbrace{\sqrt{\frac{p^{(b)}}{\mathcal{U}}} \boldsymbol{w}_{X_{k^{*}}^{l^{*}, Y_{l^{*}}}}^{T} \boldsymbol{G}_{Y_{l^{*}, Y_{l^{*}}}} \boldsymbol{V}_{Y_{l^{*}}} \boldsymbol{s}_{Y_{l^{*}}}}_{\text {self-interference, } s i \rightarrow u}+\underbrace{\boldsymbol{w}_{X_{k^{*}}^{l^{*}, Y_{l^{*}}}}^{T} \boldsymbol{\eta}_{Y_{l^{*}}}}_{\text {noise }}
\end{aligned}
$$

where $\boldsymbol{\eta}_{Y_{l^{*}}} \in \mathcal{C}^{N^{r} \times 1}$ is the circularly-symmetric complex AWGN vector with mean zero and covariance matrix $\sigma^{2} \mathbf{I}_{N^{r}}$.

The multi-user interference in the DL can be eliminated using linear ZF precoding. In FD cellular evnionments, however, SI can severely limit performance particularly in the UL. Hence, in this work, we incorporate a linear ZF-SIN precoding scheme where each BS utilizes its large scale transmit antenna array to jointly suppress multi-user interference in the DL and SI at its receiving antennas. This can be achieved at the $l$ th BS (with $N^{t} \geq N^{r}+\mathcal{U}$ ) by setting the column vectors of $V_{Y_{l}}$ to be equal to the normalized columns of $\hat{G}_{Y_{l}}^{+}=$ $\hat{\boldsymbol{G}}_{Y_{l}}^{\dagger}\left(\hat{\boldsymbol{G}}_{Y_{l}} \hat{\boldsymbol{G}}_{Y_{l}}^{\dagger}\right)^{-1}=\left[\hat{\boldsymbol{g}}_{Y_{l}, X_{k}^{l}}\right]_{1 \leq k \leq \mathcal{U}} \in \mathcal{C}^{N^{t} \times \mathcal{U}}$ where $\hat{\boldsymbol{G}}_{Y_{l}}=$ $\boldsymbol{G}_{Y_{l}}\left(\mathbf{I}_{N^{t}}-\boldsymbol{G}_{Y_{l}, Y_{l}}^{\dagger}\left(\boldsymbol{G}_{Y_{l}, Y_{l}} \boldsymbol{G}_{Y_{l}, Y_{l}}^{\dagger}\right)^{-1} \boldsymbol{G}_{Y_{l}, Y_{l}}\right)$. It should be noted that the proposed alignment-based precoder differs from the extended ZF scheme in [10] and subsequent works where 'allzero' streams are transmitted for SI cancellation purposes. In addition, the linear $\mathrm{ZF}$ decoder at the $l$-th $\mathrm{BS}$, which eliminates multi-user interference in the UL, is constructed with the normalized rows of $\boldsymbol{H}_{Y_{l}}^{+}=\left(\boldsymbol{H}_{Y_{l}}^{\dagger} \boldsymbol{H}_{Y_{l}}\right)^{-1} \boldsymbol{H}_{Y_{l}}^{\dagger}=$ $\left[\hat{\boldsymbol{h}}_{X_{k}^{l}, Y_{l}}^{T}\right]_{1 \leq k \leq \mathcal{U}}^{T} \in \mathcal{C}^{\mathcal{U} \times N^{r}}$ set as the row vectors of $\boldsymbol{W}_{Y_{l}}$.

\section{E. Intended and Interfering Signals Statistics}

We proceed by formulating the received signal-tointerference-plus-noise ratio (SINR) at the reference UE using [12]

$$
\gamma_{d}=\frac{\mathcal{X}_{d}}{\mathcal{I}_{d \rightarrow d}+\mathcal{I}_{u \rightarrow d}+\mathcal{I}_{s i \rightarrow d}+\sigma^{2}}
$$

where

$$
\begin{aligned}
& \mathcal{X}_{d}=\frac{p^{(b)}}{\mathcal{U}} d_{Y_{l^{*}}, X_{k^{*}}^{l^{*}}}^{-\beta} G_{Y_{l^{*}}, X_{k^{*}}^{l^{*}}} \\
& \mathcal{I}_{d \rightarrow d}=\frac{p^{(b)}}{\mathcal{U}} \sum_{Y_{l} \in \Phi^{(b)} \backslash\left\{Y_{l^{*}}\right\}} d_{Y_{l}, X_{k^{*}}^{l^{*}}}^{-\beta} G_{Y_{l}, X_{k^{*}}^{l^{*}}}, \\
& \mathcal{I}_{u \rightarrow d}=\sum_{X_{k}^{l} \in \tilde{\Phi}^{(u)} \backslash\left\{X_{k^{*}}^{l^{*}}\right\}} p_{X_{k}^{l}}^{(u)} d_{X_{k}^{l}, X_{k^{*}}^{l^{*}}}^{-\beta} H_{X_{k}^{l}, X_{k^{*}}^{l^{*}}}, \\
& \mathcal{I}_{s i \rightarrow d}=p_{X_{k^{*}}^{l^{*}}}^{(u)} H_{X_{k^{*}}^{l^{*}}, X_{k^{*}}^{l^{*}}},
\end{aligned}
$$

linear precoder is selected in the direction of the projection of $\boldsymbol{g}_{Y_{l^{*}}, X_{k^{*}}^{l^{*}}}$ on the $\operatorname{Null}\left(\left[\boldsymbol{g}_{Y_{l^{*}}, X_{k}^{l^{*}}}\right]_{1 \leq k \leq \mathcal{U}, k \neq k^{*}}, \boldsymbol{G}_{Y_{l^{*}}, Y_{l^{*}}}\right)$ of dimension $D_{d}=N^{t}-N^{r}-\mathcal{U}+1$, i.e., $\boldsymbol{v}_{Y_{l^{*}}, X_{k^{*}}^{l^{*}}}=\frac{\hat{\boldsymbol{g}}_{Y_{l^{*}, X_{k^{*}}}}}{\left\|\hat{\boldsymbol{g}}_{Y_{l^{*}}, X_{k^{*}}^{l^{*}}}\right\|}$. Therefore, considering isotropic Rayleigh fading links, we have $G_{Y_{l^{*}}, X_{k^{*}} *} \sim \operatorname{Gamma}\left(D_{d}, 1\right)$. Under the assumption that the column vectors of $V_{Y_{l}}$ are independent, their squared norm is a unit-mean exponential random variable resulting in $G_{Y_{l}, X_{k^{*}} *} \sim \operatorname{Gamma}(\mathcal{U}, 1)$. Moreover, $H_{X_{k}^{l}, X_{k^{*}}^{l^{*}}} \sim$ $\operatorname{Gamma}(1,1)$. The SI channel power gain, $H_{X_{k^{*}}, X_{h^{*}}^{l^{*}}}$, on the other hand, is a non-central Chi-squared random variable with the following p.d.f. and m.g.f.

$$
\begin{aligned}
\mathcal{P}_{H_{(.)}}(h) & =\frac{1+K}{\Omega} \exp \left(-\left(K+\frac{(1+K) h}{\Omega}\right)\right) \\
& \times I_{0}\left(2 \sqrt{\frac{K(1+K) h}{\Omega}}\right)
\end{aligned}
$$

and

$$
\mathcal{M}_{H_{(\cdot)}}(z)=\frac{1+K}{1+K+\Omega z} \exp \left(-\frac{K \Omega z}{1+K+\Omega z}\right)
$$

where $K=\frac{\mu^{2}}{2 v^{2}}$ and $\Omega=\mu^{2}+2 v^{2}$ are respectively the Rician factor (ratio of dominant over scattered paths power) and fading attenuation (channel variance). For completeness, similar expressions for a Gamma-distributed random variable $G_{(.)}$with shape parameter $\kappa$ and scale parameter $\theta$ are respectively given by

$$
\mathcal{P}_{G_{(.)}}(g)=\frac{g^{\kappa-1}}{\theta^{\kappa} \Gamma(\kappa)} \exp \left(-\frac{g}{\theta}\right)
$$

and

$$
\mathcal{M}_{G_{(.)}}(z)=\frac{1}{(1+\theta z)^{\kappa}} .
$$

Next, we can express the received SINR from the reference $\mathrm{UE}$ at its serving BS as follows

$$
\gamma_{u}=\frac{\mathcal{X}_{u}}{\mathcal{I}_{u \rightarrow u}+\mathcal{I}_{d \rightarrow u}+\sigma^{2}}
$$

where

$$
\begin{gathered}
\mathcal{X}_{u}=p_{X_{k^{*}}^{l^{*}}}^{(u)} d_{X_{k^{*}}^{l^{*}}, Y_{l^{*}}}^{-\beta} H_{X_{k^{*}}^{l^{*}}, Y_{l^{*}}}, \\
\mathcal{I}_{u \rightarrow u}=\sum_{X_{k}^{l} \in \tilde{\Phi}(u) \backslash\left\{\forall X_{k}^{l^{*}}\right\}} p_{X_{k}^{l}}^{(u)} d_{X_{k}^{l}, Y_{l^{*}}}^{-\beta} H_{X_{k}^{l}, Y_{l^{*}}}, \\
\mathcal{I}_{d \rightarrow u}=\frac{p^{(b)}}{\mathcal{U}} \sum_{Y_{l} \in \Phi^{(b)} \backslash\left\{Y_{l^{*}}\right\}} d_{Y_{l}, Y_{l^{*}}}^{-\beta} G_{Y_{l}, Y_{l^{*}}}, \\
H_{X_{k^{*}}^{l^{*}}, Y_{l^{*}}} \triangleq\left|\boldsymbol{w}_{X_{k^{*}}^{l^{*}}, Y_{l^{*}}} \boldsymbol{h}_{X_{k^{*}}^{l^{*}}, Y_{l^{*}}}\right|^{2}, H_{X_{k}^{l}, Y_{l^{*}}} \triangleq\left|\boldsymbol{w}_{X_{k^{*}}^{l^{*}}, Y_{l^{*}}} \boldsymbol{h}_{X_{k}, Y_{l^{*}}}\right|^{2}, \text { and } G_{Y_{l}, Y_{l^{*}}} \triangleq\left\|\boldsymbol{w}_{X_{k^{*}}^{l^{*}}, Y_{l^{*}}} \boldsymbol{G}_{Y_{l}, Y_{l^{*}}} \boldsymbol{V}_{Y_{l}}\right\|^{2} \text {. We can } \\
\text { further deduce using assumptions mentioned previously that } \\
H_{X_{k^{*}}^{l^{*}}, Y_{l^{*}}} \sim \operatorname{Gamma}\left(D_{u}, 1\right), \text { where } D_{u}=N^{r}-\mathcal{U}+1, \\
H_{X_{k}^{l}, Y_{l^{*}}} \sim \operatorname{Gamma}(1,1), \text { and } G_{Y_{l}, Y_{l^{*}}} \sim \operatorname{Gamma}(\mathcal{U}, 1) .
\end{gathered}
$$




\section{F. Self-Interference-Aware Power Control}

The proposed UL SIA fractional power control mechanism can be described as

$$
p_{X_{k}^{l}}^{(u)}=\min \left(p_{0} d_{X_{k}^{l}, Y_{l}}^{\psi \beta}, H_{X_{k}^{l}, X_{k}^{l}}^{-1} I_{\mathrm{SI}}, p^{(u)}\right)
$$

where $p_{0}, \psi(\in[0,1]), I_{\mathrm{SI}}$, and $p^{(u)}$ are respectively the normalized power density, compensation factor, tolerable SI level, and maximum available transmit power at the UE [13], [14]. The value of $I_{\mathrm{SI}}$ in practice can be set according to the difference in the noise floor power from the SI cancellation gain. The c.d.f. of the transmit power can be expressed as

$$
\begin{aligned}
\mathcal{F}_{p_{X_{k}^{(u)}}^{(u)}}(x) & =\left(1-\mathcal{H}\left(x-p^{(u)}\right)\right) \mathscr{P}\left(\hat{p}_{X_{k}^{l}}^{(u)} \leq x\right) \\
& +\mathcal{H}\left(x-p^{(u)}\right)
\end{aligned}
$$

where $\hat{p}_{X_{k}^{l}}^{(u)}=\min \left(p_{0} d_{X_{k}^{l}, Y_{l}}^{\psi \beta}, H_{X_{k}^{l}, X_{k}^{l}}^{-1} I_{\mathrm{SI}}\right)$. We proceed by deriving the c.d.f. expression

$$
\mathcal{F}_{\hat{p}_{X_{k}^{l}}^{(u)}}(x)=1-\Xi_{I}(x)\left(1-\Xi_{I I}(x)\right)
$$

where

$$
\Xi_{I}(x)=\exp \left(-\pi \lambda^{(b)}\left(\frac{x}{p_{0}}\right)^{\frac{2}{\psi \beta}}\right)
$$

and

$$
\Xi_{I I}(x)=Q_{1}\left(\sqrt{2 K}, \sqrt{\frac{2(1+K) I_{\mathrm{SI}}}{\Omega x}}\right) .
$$

With further analysis, an expression for the p.d.f. of the SIA transmit power at the UE side can be obtained as in (23).

\section{Spectral EfFiciency Analysis}

We can formulate the DL and UL spectral efficiencies (in $\mathrm{b} / \mathrm{s} / \mathrm{Hz}$ ) of the FD massive MIMO cellular network as in (24) and (25) [15]. The conditional statistics of the intended signal power in the DL and UL are respectively given by

$$
\mathcal{M}_{\mathcal{X}_{d} \mid r}(z)=\left(1+\frac{p^{(b)} z}{\mathcal{U} r^{\beta}}\right)^{-D_{d}}
$$

and

$$
\mathcal{M}_{\mathcal{X}_{u} \mid p, r}(z)=\left(1+\frac{p z}{r^{\beta}}\right)^{-D_{u}} .
$$

Finally, we proceed by developing closed-form expressions for the conditional statistics of the different interference terms as in (29)-(32). The proofs - omitted due to space limitations follow from a similar methodology to that in [16].

$$
\begin{aligned}
& \mathcal{P}_{p_{X_{k}^{l}}^{(u)}}(x)= \begin{cases}\delta\left(x-p^{(u)}\right) \Xi_{I}\left(p^{(u)}\right)\left(1-\Xi_{I I}\left(p^{(u)}\right)\right) & x \geq p^{(u)} \\
\frac{(1+K) I_{\mathrm{SI}}}{\Omega x^{2}} \Xi_{I}(x) \exp \left(-\left(\frac{(1+K) I_{\mathrm{SI}}}{\Omega x}+K\right)\right){ }_{0} \tilde{F}_{1}\left(; 1 ; \frac{K(1+K) I_{\mathrm{SI}}}{\Omega x}\right) & \\
+\frac{2 \pi \lambda^{(b)}}{\psi \beta p_{0}}\left(\frac{x}{p_{0}}\right)^{\frac{2}{\beta \psi}} \Xi_{I}(x)\left(1-\Xi_{I I}(x)\right) & x<p^{(u)}\end{cases} \\
& \mathcal{S}_{d}=\mathbb{E}\left\{\log _{2}\left(1+\gamma_{d}\right)\right\}=\log _{2}(e) \int_{0}^{+\infty} \int_{0}^{+\infty} \mathcal{M}_{\mathcal{I}_{s i \rightarrow d} \mid p}(z) \mathcal{M}_{\mathcal{I}_{u \rightarrow d} \mid p}(z) \int_{0}^{+\infty}\left(1-\mathcal{M}_{\mathcal{X}_{d} \mid r}(z)\right) \mathcal{M}_{\mathcal{I}_{d \rightarrow d} \mid r}(z) \frac{\exp \left(-\sigma^{2} z\right)}{z} \\
& \times \mathcal{P}_{p_{X_{k}^{(u)}}^{(u)}}(p) \mathcal{P}_{d_{Y_{l^{*}, X^{\prime}}^{l^{*}}}}(r) \mathrm{d} z \mathrm{~d} p \mathrm{~d} r \\
& \mathcal{S}_{u}=\mathbb{E}\left\{\log _{2}\left(1+\gamma_{u}\right)\right\}=\log _{2}(e) \int_{0}^{+\infty} \mathcal{M}_{\mathcal{I}_{d \rightarrow u}}(z) \int_{0}^{+\infty} \int_{0}^{+\infty}\left(1-\mathcal{M}_{\mathcal{X}_{u} \mid p, r}(z)\right) \mathcal{M}_{\mathcal{I}_{u \rightarrow u} \mid p, r}(z) \frac{\exp \left(-\sigma^{2} z\right)}{z}
\end{aligned}
$$

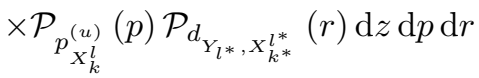

$$
\begin{aligned}
& \mathcal{M}_{\mathcal{I}_{d \rightarrow d} \mid r}(z)=\exp \left(-\pi \lambda^{(b)}\left[r^{2}\left(\left(1+\frac{p^{(b)} z}{\mathcal{U} r^{\beta}}\right)^{-\mathcal{U}}-1\right)+\frac{\Gamma\left(\mathcal{U}+\frac{2}{\beta}\right)}{\Gamma(\mathcal{U})\left(\frac{p^{(b)} z}{\mathcal{U}}\right)^{\mathcal{U}}}\left(\Gamma\left(1-\frac{2}{\beta}\right)\left(\frac{p^{(b)} z}{\mathcal{U}}\right)^{\mathcal{U}+\frac{2}{\beta}}\right.\right.\right. \\
& \left.\left.\left.-\mathcal{U} \Gamma(\mathcal{U}) r^{\mathcal{U} \beta+2}{ }_{2} \tilde{F}_{1}\left(1+\mathcal{U}, \mathcal{U}+\frac{2}{\beta} ; 1+\mathcal{U}+\frac{2}{\beta} ;-\frac{\mathcal{U} r^{\beta}}{p^{(b)} z}\right)\right)\right]\right) \\
& \mathcal{M}_{\mathcal{I}_{u \rightarrow u} \mid p, r}=\exp \left(-\pi \mathcal{U} \lambda^{(b)}\left[\Gamma\left(1-\frac{2}{\beta}\right) \Gamma\left(1+\frac{2}{\beta}\right)(p z)^{\frac{2}{\beta}}-r^{2}\left(1-\frac{2 r^{\beta}}{(2+\beta) p z}{ }_{2} F_{1}\left(1,1+\frac{2}{\beta} ; 2+\frac{2}{\beta} ;-\frac{r^{\beta}}{p z}\right)\right)\right]\right)
\end{aligned}
$$




$$
\mathcal{M}_{\mathcal{I}_{u \rightarrow d} \mid p}(z)=\exp \left(-\pi \mathcal{U} \lambda^{(b)}(p z)^{\frac{2}{\beta}} \Gamma\left(1-\frac{2}{\beta}\right) \Gamma\left(1+\frac{2}{\beta}\right)\right)
$$

$$
\mathcal{M}_{\mathcal{I}_{d \rightarrow u}}(z)=\exp \left(-\pi \lambda^{(b)}\left(\frac{p^{(b)} z}{\mathcal{U}}\right)^{\frac{2}{\beta}} \frac{\Gamma\left(1-\frac{2}{\beta}\right) \Gamma\left(\mathcal{U}+\frac{2}{\beta}\right)}{\mathcal{U}^{\frac{2}{\beta}} \Gamma(\mathcal{U})}\right)
$$

$$
\mathcal{M}_{\mathcal{I}_{s i \rightarrow d} \mid p}(z)=\frac{1+K}{1+K+\Omega p z} \exp \left(-\frac{K \Omega p z}{1+K+\Omega p z}\right)
$$

\section{Performance Analysis}

In this section, we provide several numerical examples in order to assess the performance of the FD massive MIMO cellular network under different system settings. The BS deployment density is set to be $\frac{4}{\pi}$ per unit area $(\mathrm{km} \times \mathrm{km})$. The total system bandwidth is considered to be $B=20 \mathrm{MHz}$. The noise power is calculated using $\sigma^{2}=-170+10 \log _{10}(B)+N_{f}$ (dBm), where $N_{f}$ is the noise figure. The maximum available transmit powers at the BSs and UEs are set to $43 \mathrm{dBm}$ and 23 $\mathrm{dBm}$, respectively. For comparison purposes, we consider the case where the FD systems simultaneously utilize the entire bandwidth for the DL and UL, whereas $B$ is divided equally between the two operations in the HD scenarios.

1) Different FD cellular setups: We compare the DL and UL performance of several different FD cellular networks, namely, LSAS (Massive MIMO), MIMO, and SISO, in Fig. 1. The results confirm prior findings that the UL rate is the main performance bottleneck in FD cellular systems with baseline single-antenna transmission. Furthermore, it can be observed that significant performance gains can be achieved by exploiting the large scale antenna arrays with linear ZFSIN precoding and linear ZF receive combining. For example, with $\beta=4$, the UL spectral efficiency in the proposed massive MIMO cellular setup is more than 96 times greater than that in the SISO case. Note that by increasing the antenna array size, further improvements can be realized from the added degrees of freedom as well as the potential to linearly reduce the transmit powers of the BSs and UEs without degrading the received signal-to-noise ratio (SNR).

2) FD versus $H D$ massive MIMO: Next, we investigate the performance of the FD massive MIMO cellular network with respect to its HD counterpart over a wide range of SI channel attenuations in Fig. 2. It can be seen that any potential improvements from the FD operation occurs for SI channel attenuations well below $-80 \mathrm{~dB}$. This trend indicates that without advanced SI mitigation solutions being available at the terminals, the conventional HD cellular LSAS is arguably the more sensible deployment choice. With nearly perfect SI cancellation at the reference UE, on the other hand, the maximum system spectral efficiency gain in the FD massive MIMO cellular network over its analogous HD variant is $42 \%$ (resulting from a $71 \%$ increase and a $6 \%$ decrease in the DL and UL spectral efficiencies, respectively).

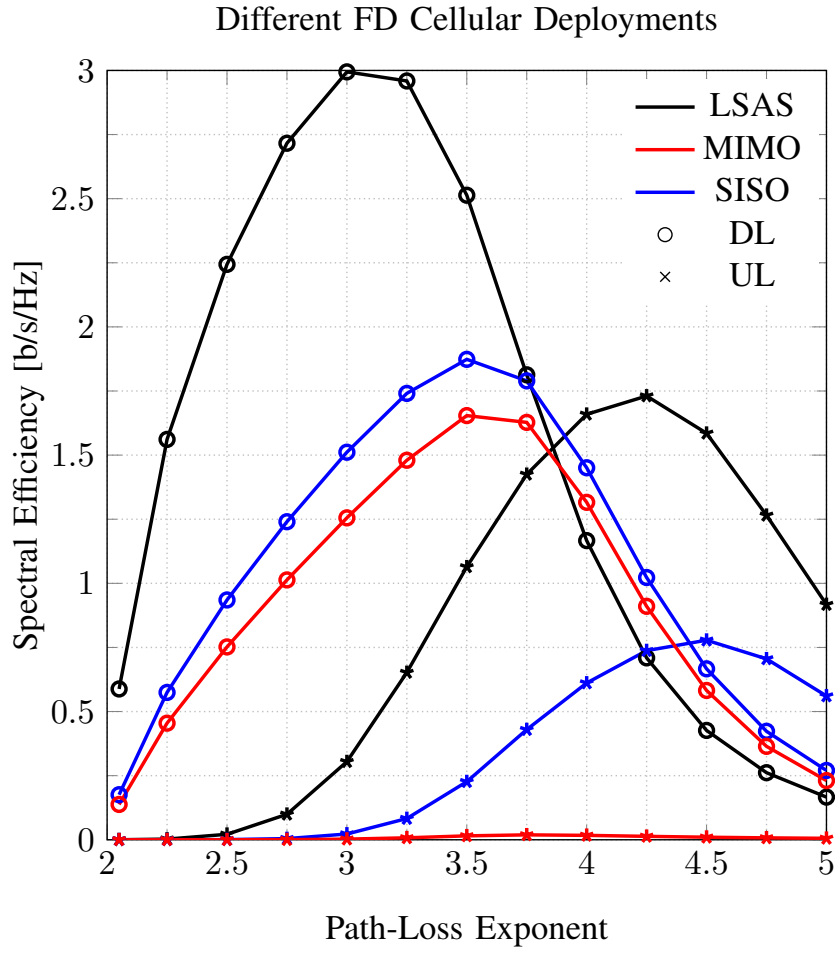

Fig. 1: System parameters are: $\lambda^{(b)}=\frac{4}{\pi} \mathrm{BSs} / \mathrm{km}^{2}$, LSAS $\left(N^{t}=80\right.$, $\left.N^{r}=20, \mathcal{U}=8, p^{(b)}=30 \mathrm{dBm}\right), \operatorname{MIMO}\left(N^{t}=16, N^{r}=8\right.$, $\left.\mathcal{U}=4, p^{(b)}=43 \mathrm{dBm}\right)$, SISO $\left(N^{t}=1, N^{r}=1, \mathcal{U}=1, p^{(b)}=43\right.$ $\mathrm{dBm}), p^{(u)}=23 \mathrm{dBm}, p_{0}=-80 \mathrm{dBm}, B=20 \mathrm{MHz}, N_{f}=10$ $\mathrm{dB}, \psi=1, \Omega=-80 \mathrm{~dB}, K=1$.

3) Uplink power control mechanisms: The results presented so far were based on the conventional UL fractional power control mechanism defined in the existing LTE standards for HD cases. Next, we study the performance of the FD massive MIMO cellular network with different fixed (at maximum power), conventional, and proposed SIA fractional power control protocols under different SI channel attenuations in Fig. 3. It can be observed that the lack of self-interference-awareness in the case of fixed as well as fractional power allocation strategies means that the DL performance significantly suffers in the case of large SI channel attenuations. The proposed scheme can therefore serve as a safe-guard mechanism for ensuring a certain maximum SI level is not exceeded. 
FD versus HD Massive MIMO

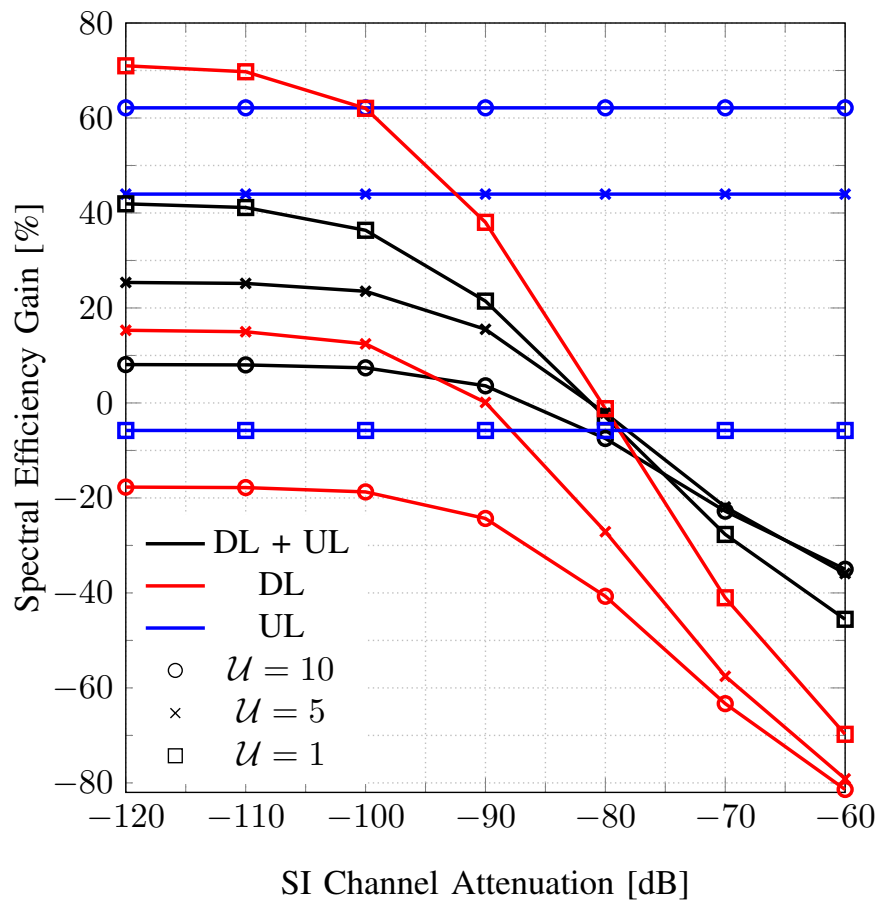

Fig. 2: System parameters are: $\lambda^{(b)}=\frac{4}{\pi} \mathrm{BSs} / \mathrm{km}^{2}, N^{t}=350, N^{r}=$ $50, p^{(b)}=30 \mathrm{dBm}, p^{(u)}=23 \mathrm{dBm}, p_{0}=-80 \mathrm{dBm}, B=20 \mathrm{MHz}$, $N_{f}=10 \mathrm{~dB}, \beta=4, \psi=1, K=1$.

\section{SUMMARY}

In this paper, we provided a stochastic geometry analysis of FD massive MIMO cellular networks. The system spectral efficiency with linear ZF-SIN precoder, SIA fractional power control, and linear ZF decoder was characterized over Rician SI and Rayleigh intended and other-interference fading channels. The results highlighted the promising potential of exploiting massive MIMO towards unlocking the UL rate bottleneck in FD cellular environments. The performance gain of the FD massive MIMO cellular network over its HD counterpart was on the other hand shown to be largely dependent on the SI cancellation capabilities of the user terminals.

\section{REFERENCES}

[1] F. Boccardi, R. W. Heath, A. Lozano, T. L. Marzetta, and P. Popovski, "Five disruptive technology directions for 5G," IEEE Commun. Mag., vol. 52, no. 2, pp. 74-80, Feb. 2014.

[2] J. Tang, D. K. C. So, E. Alsusa, K. A. Hamdi, and A. Shojaeifard, "Resource allocation for energy efficiency optimization in heterogeneous networks," IEEE J. Sel. Areas Commun., vol. 33, no. 10, pp. 2104-2117, Oct. 2015.

[3] P. Patcharamaneepakorn, S. Wu, C. X. Wang, H. Aggoune, M. Alwakeel, $\mathrm{X}$. Ge, and M. Di Renzo, "Spectral, energy and economic efficiency of 5G multi-cell massive MIMO systems with generalized spatial modulation," IEEE Trans. Veh. Technol., accepted 2016.

[4] M. Duarte, C. Dick, and A. Sabharwal, "Experiment-driven characterization of full-duplex wireless systems," IEEE Trans. Wireless Commun., vol. 11, no. 12, pp. 4296-4307, Dec. 2012.

[5] M. Chung, M. S. Sim, J. Kim, D. K. Kim, and C. b. Chae, "Prototyping real-time full duplex radios," IEEE Commun. Mag., vol. 53, no. 9, pp. 56-63, Sep. 2015.

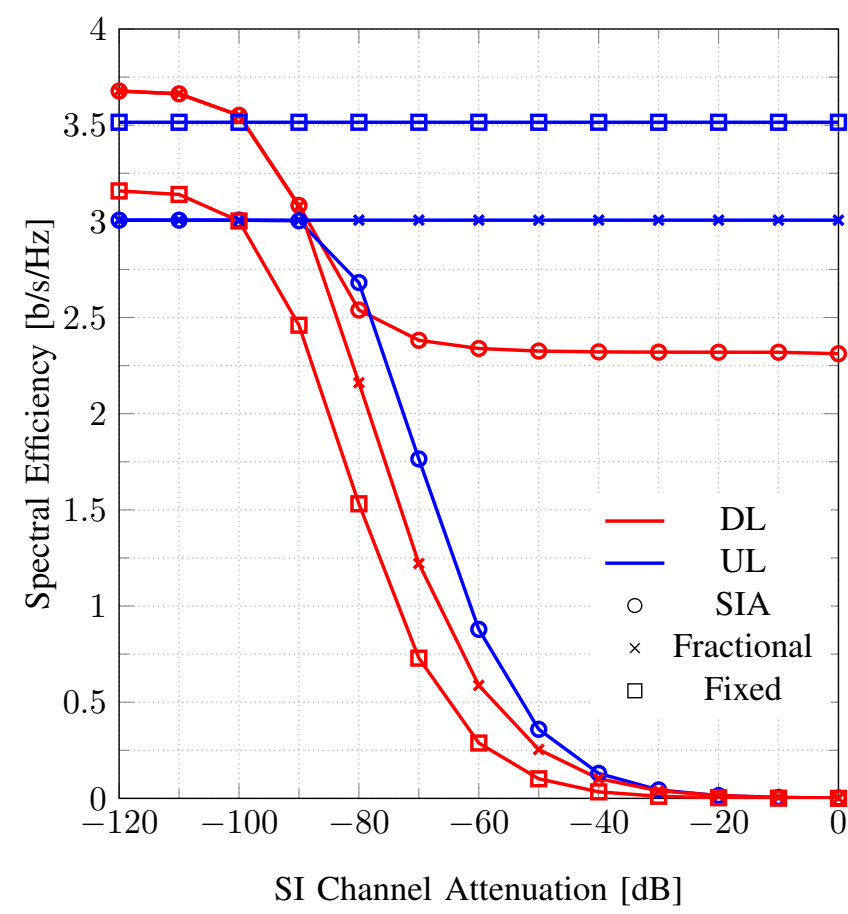

Fig. 3: System parameters are: $\lambda^{(b)}=\frac{4}{\pi} \mathrm{BSs} / \mathrm{km}^{2}, N^{t}=150, N^{r}=$ $50, \mathcal{U}=4, p^{(b)}=30 \mathrm{dBm}, p^{(u)}=23 \mathrm{dBm}, p_{0}=-80 \mathrm{dBm}$, $\frac{I_{\mathrm{SI}}}{\sigma^{2}}=25 \mathrm{~dB}, p_{f}^{(u)}=23 \mathrm{dBm}, B=20 \mathrm{MHz}, N_{f}=10 \mathrm{~dB}, \beta=4$, $\stackrel{\sigma^{2}}{\psi}=1, K=1$.

[6] I. Atzeni and M. Kountouris, "Full-duplex MIMO small-cell networks: Performance analysis," CoRR, vol. abs/1504.04167, 2015.

[7] M. Mohammadi, H. A. Suraweera, and C. Tellambura, "Full-duplex cloud-RAN with uplink/downlink remote radio head association," CoRR, vol. abs/1602.08836, 2016.

[8] A. Sadeghi, M. Luvisotto, F. Lahouti, S. Vitturi, and M. Zorzi, "Statistical QoS analysis of full duplex and half duplex heterogeneous cellular networks," ArXiv e-prints, Apr. 2016.

[9] H. ElSawy, A. AlAmmouri, O. Amin, and M.-S. Alouini, "Can uplink transmissions survive in full-duplex cellular environments?" ArXiv eprints, Apr. 2016.

[10] B. Yin, M. Wu, C. Studer, J. R. Cavallaro, and J. Lilleberg, "Full-duplex in large-scale wireless systems," in Proc. Asilomar Conf. Signals, Syst. and Comput. (ASILOMAR'13), Nov. 2013, pp. 1623-1627.

[11] K. Min, S. Park, Y. Jang, T. Kim, and S. Choi, "Antenna ratio for sum-rate maximization in full-duplex large-array base station with halfduplex multi-antenna users," IEEE Trans. Veh. Technol., accepted 2016.

[12] A. Shojaeifard, K. A. Hamdi, E. Alsusa, D. K. C. So, and J. Tang, "Exact SINR statistics in the presence of heterogeneous interferers," IEEE Trans. Inform. Theory, vol. 61, no. 12, pp. 6759-6773, Dec. 2015.

[13] M. D. Renzo and P. Guan, "Stochastic geometry modeling and systemlevel analysis of uplink heterogeneous cellular networks with multiantenna base stations," IEEE Trans. Commun., vol. 64, no. 6, pp. 24532476, June 2016.

[14] M. R. A. Khandaker and Y. Rong, "Interference MIMO relay channel: Joint power control and transceiver-relay beamforming," IEEE Trans, Signal Process., vol. 60, no. 12, pp. 6509-6518, Dec. 2012.

[15] A. Shojaeifard, K. A. Hamdi, E. Alsusa, D. K. C. So, and J. Tang, "A unified model for the design and analysis of spatially-correlated loadaware HetNets," IEEE Trans. Commun., vol. 62, no. 11, pp. 1-16, Nov. 2014.

[16] A. Shojaeifard, K. A. Hamdi, E. Alsusa, D. K. C. So, J. Tang, and K. K. Wong, "Design, modeling, and performance analysis of multi-antenna heterogeneous cellular networks," IEEE Trans. Commun., vol. 64, no. 7, pp. 3104-3118, July 2016. 